\title{
Hábitos alimentares e de higiene no período de isolamento social durante a pandemia da Covid-19 no Brasil
}

\author{
Food and hygiene habits in the period of social isolation during the Covid-19 pandemic in Brazil \\ Hábitos alimentarios e higiénicos en el periodo de aislamiento social durante la pandemia del
}

Covid-19 en el Brasil

Recebido: 26/04/2021 | Revisado: 05/05/2021 | Aceito: 09/05/2021 | Publicado: 23/05/2021

Sandro Dalmolin

ORCID: https://orcid.org/0000-0003-3358-1377 Universidade Estadual do Rio Grande do Sul, Brasil E-mail: sandrodalmolin@gmail.com

Magnólia Martins Erhardt

ORCID: https://orcid.org/0000-0003-3730-2515 Universidade Estadual do Rio Grande do Sul, Brasil E-mail: magnolia-erhardt@uergs.edu.br

Jeferson Aloísio Ströher

ORCID: https://orcid.org/0000-0002-5314-9269 Universidade Estadual do Rio Grande do Sul, Brasil E-mail: jeferson.stroher@hotmail.com

Hans Fröder

ORCID: https://orcid.org/0000-0003-1551-236X Universidade Estadual do Rio Grande do Sul, Brasil E-mail: hfroder@univates.br

Marion Ruis

ORCID: https://orcid.org/0000-0001-8920-2378 Universidade Estadual do Rio Grande do Sul, Brasil E-mail: marionruis@univates.br

Neila Silvia Pereira dos Santos Richards

ORCID: https://orcid.org/0000-0001-6610-5567

Universidade Federal de Santa Maria, Brasil E-mail: neilarichardsprof@gmail.com

Valéria Pinheiro Braccini

ORCID: https://orcid.org/0000-0002-6891-0196

Universidade Federal de Santa Maria, Brasil E-mail: valurug@gmail.com

Maximiliano Segundo Escalona Jiménez

ORCID: https://orcid.org/0000-0002-2516-4330

Universidade Federal de Santa Maria, Brasil

E-mail: maximiliano.escalonaj@gmail.com

\begin{abstract}
Resumo
Esse trabalho objetivou analisar, mediante pesquisa totalmente on-line, os hábitos alimentares e de higiene pessoal para combater o coronavírus, no período de isolamento social imposto pelo governo, durante a pandemia da Covid-19. Elaborou-se um questionário, o qual foi enviado a contatos por meio das redes sociais WhatsApp ${ }^{\circledR}$ e Messenger ${ }^{\circledR}$, com objetivo de descobrir se os participantes estavam fazendo uso das etiquetas comportamentais recomendadas pela ANVISA para combater a Covid-19; verificar se as pessoas estavam fazendo uso da máscara facial e respeitando o distanciamento social; avaliar os impactos na rotina das pessoas, tanto na alimentação como nos serviços de alimentação que utilizavam antes do isolamento; identificar como estavam adquirindo os alimentos e quais serviços de alimentação fizeram mais falta. A pesquisa teve a participação de pessoas de todas as regiões do estado do Rio Grande do Sul, bem como de outros estados do país. Observou-se, mediante os resultados obtidos que, apesar da maciça divulgação, ainda há a necessidade de conscientização quanto ao uso da máscara. Constatou-se que a maioria dos pesquisados não apresentavam sintomas e não necessitavam de isolamento; as compras ainda continuaram sendo feitas no modo tradicional, indo ao mercado, o que pode tornar-se ponto disseminador da doença. Sugere-se que sejam intensificadas as campanhas de orientação, bem como a realização de novas pesquisas que justifiquem intensificar medidas de etiquetas sanitárias e maior conscientização coletiva.
\end{abstract}

Palavras-chave: Hábitos alimentares; Higiene de alimentos; Covid-19; Isolamento social. 


\begin{abstract}
This work aimed to analyze, through an entirely on line survey, the eating and personal hygiene habits to combat the Coronavirus during the period of social isolation imposed by the government during the covid-19 pandemic. A questionnaire was prepared and contacts were sent through social networks, Wattsapp® and Messenger® with the objective of finding out if the participants are making use of the behavioral labels recommended by ANVISA to combat covid-19; to verify if people are making use of the face mask and respecting social distancing; to evaluate the impacts on people's routine, both in their eating habits and in the food services they used before the isolation; how they are acquiring their food; which food services were missed the most. The survey had the participation of people from all regions of the state of Rio Grande do Sul as well as from other states in the country. The results obtained show that, despite the massive publicity, the use of the mask still needs a lot of work to raise awareness; the vast majority of the people surveyed had no symptoms and did not need isolation; purchases are still made in the traditional way by going to the market, which can become a disseminating point of the disease. It is suggested that orientation campaigns be intensified as well as new research to justify the intensification of sanitary label measures and greater collective awareness so that we can get out of this pandemic without losing more lives.
\end{abstract}

Keywords: Eating habits; Food hygiene; Covid-19; Social isolation.

\title{
Resumen
}

Este trabajo tuvo como objetivo analizar, a través de una encuesta totalmente on line, los hábitos alimenticios y de higiene personal para combatir el Coronavirus durante el período de aislamiento social impuesto por el gobierno durante la pandemia del covid-19. Se elaboró un cuestionario y se enviaron contactos a través de las redes sociales, Wattsapp ${ }^{\circledR}$ y Messenger® con el objetivo de conocer si los participantes están haciendo uso de las etiquetas de comportamiento recomendadas por ANVISA para combatir el covid-19; verificar si las personas están haciendo uso de la mascarilla y respetando el distanciamiento social; evaluar los impactos en la rutina de las personas, tanto en sus hábitos alimenticios como en los servicios de alimentación que utilizaban antes del aislamiento; cómo están adquiriendo sus alimentos; cuáles son los servicios de alimentación que más echaron de menos. La encuesta contó con la participación de personas de todas las regiones del estado de Rio Grande do Sul, así como de otros estados del país. Los resultados obtenidos muestran que, a pesar de la publicidad masiva, el uso de la mascarilla aún necesita mucho trabajo de concientización; la gran mayoría de las personas encuestadas no tenía síntomas y no necesitaba el aislamiento; las compras aún se realizan de manera tradicional yendo al mercado, lo que puede convertirse en un punto de diseminación de la enfermedad. Se sugiere intensificar las campañas de orientación, así como nuevas investigaciones que justifiquen la intensificación de las medidas de la etiqueta sanitaria y una mayor concienciación colectiva para poder salir de esta pandemia sin perder más vidas.

Palabras clave: Hábitos alimenticios; Higiene de los alimentos; Covid-19; Aislamiento social.

\section{Introdução}

Sair de casa tornou-se algo perigoso, jantares aos finais de semana ficaram cada vez mais raros e ir ao supermercado converteu-se em um desafio para população em geral. Para evitar que o vírus da Covid-19 se proliferasse, várias medidas foram tomadas entre a população, como o isolamento social, em que o governo orientou as pessoas a não saírem de casa. O objetivo é evitar aglomerações, a fim de manter distância mínima de um metro e meio por pessoas (Farias, 2020). O isolamento social é uma estratégia defendida pela Organização Mundial de Saúde (OMS), no sentido de diminuir a curva de crescimento de contaminação pelo novo coronavírus, evitando quea população fique infectada ao mesmo tempo e sobrecarregue os sistemas de saúde (Pereira et al., 2020). Ademais, o uso obrigatório da máscara facial e a utilização de álcool em gel tornaram-se comuns no dia a dia.

Em relação aos hábitos alimentares, verificou-se se as pessoas estavam preocupadas com a alimentação saudável ou houve aumento de consumo de produtos de alto valor calórico. Intentou-se, também, averiguar como as pessoas participantes adquiriram os alimentos e quais métodos foram utilizados para higienizá-los, uma vez que a relação com os alimentos também mudou. As compras nos mercados passaram a ser desafiadoras, pois são focos de contaminação. É preciso ir direto ao ponto e comprar apenas o necessário (Guenther, 2020).

Reitera-se que esta pesquisa foi realizada inteiramente de forma on-line, cujo questionário foi enviado através das redes sociais. Diante do exposto, informa-se que esta investigação se torna muito relevante, já que buscou descobrir como as pessoas reagiram diante de um cenário totalmente diferente das rotinas. 


\subsection{Um breve histórico sobre a evolução da Covid-19}

No final de dezembro de 2019, a China comunicou à Organização Mundial da Saúde (OMS) o primeiro caso de Covid-19, ocorrido no município de Wuham. A doença Covid-19 (Coronavírus Disease 2019) é uma infecção respiratória provocada pelo Coronavírus da Síndrome Respiratória Aguda Grave 2 (SARS-CoV-2) (Schuchmann, et al., 2020). Rapidamente o novo coronavírus se espalhou pelo mundo, com casos relatados em 19 países no final de janeiro de 2020. Em 11 de março de 2020, a OMS declarou a Covid-19 como uma pandemia (Schmidt et al., 2020). Nas semanas seguintes, o número de casos de Covid-19 fora da China aumentou 13 vezes e a quantidade de países afetados triplicou. São muitas pessoas infectadas em 114 nações, sendo que 4.291 pessoas morreram (Universidade Aberta do Sistema Único de Saúde [UNA-SUS], 2020).

No dia três de abril de 2020, 206 países registravam infecções pelo novo coronavírus, com total de 976.249 casos confirmados e 50.489 mortes, de acordo com a OMS (Ministério da Saúde, 2020).

No Brasil, a Emergência em Saúde Pública de Importância Nacional (ESPIN), em decorrência da infecção humana pelo novo coronavírus, foi declarada em fevereiro de 2020, por meio da Portaria no 188/20 (Brasil, 2020). No dia 4 de fevereiro, o presidente da República Jair Messias Bolsonaro declarou estado de emergência, e o governo passou a planejar a operação de resgate. Isso exigia colocar militares na área de risco e trazer pessoas com potencial infecção para o solo brasileiro, uma ação arriscada (Folha de São Paulo, 2020). Em abril, o Brasil contava com 9.056 casos confirmados e 359 mortes (Ministério da Saúde, 2020).

Em janeiro de 2021, o país registrava total de 196.591 óbitos desde o começo da pandemia. Sobre os casos confirmados, 7.754.560 brasileiros já tiveram ou têm o novo coronavírus, com 22.489 destes confirmados somente em um dia (Portal G1, 2021). O balanço mostra ainda que 6.813.008 pessoas se recuperaram da Covid-19. Os dados divulgados pelo Ministério da Saúde vêm de informações levantadas pelas Secretarias Estaduais de Saúde de todo o país (Brandão, 2021).

O mundo registrou mais de 1,1 milhão de novos casos de Covid-19, nos primeiros dois dias de 2021, segundo levantamento da Universidade Johns Hopkins. Os números foram coletados por Estados Unidos, Reino Unido, Rússia, Brasil, Itália e Índia,que lideraram o ranking de novos casos (Portal G1, 2021). Ainda de acordo com Portal G1 (2021), até o momento, a Covid-19 deixou mais de 1,8 milhão de vítimas no mundo.

\subsection{Medidas de controle para evitar a proliferação do vírus}

Desde a emergência, na China, em dezembro de 2019, do novo coronavírus, a humanidade tem enfrentado uma grave crise sanitária global, muitas das medidas de controle da pandemia foram introduzidas ao mesmo tempo e tiveram graus variados de adesão nos diferentes países (Aquino et al., 2020). Em um contexto em que não se dispõem de recursos farmacêuticos para conter o avanço do coronavírus em todo o mundo, o distanciamento social ocupa posição de centralidade nas ações de mitigação da Covid-19 (G. L. A. Oliveira et al. 2020).

Em casos extremos é adotado o Isolamento Social (IS), conceitualmente, quando as pessoas não podem sair de suas casas como forma de evitar a proliferação do vírus. Dessa forma, há ainda a recomendação de que as pessoas suspeitas de portarem o vírus permaneçam em quarentena por quatorze dias, pois este é o período de incubação do SARS-CoV2, ou seja, o tempo para o vírus manifestar-se no corpo do indivíduo (Pereira et al., 2020, p.4).

Conforme descrito na NT n 47 de 03/06/2020 (Agência Nacional de Vigilância Sanitária [ANVISA], 2020) sobre o distanciamento: a dinâmica da pandemia do novo coronavírus mostra que a transmissãodo vírus tem ocorrido de pessoa para pessoa, pelo contato próximo com um indivíduo infectado ou por contágio indireto, ou seja, por meio de superfícies e objetos contaminados, principalmente pela tosse e espirro de pessoas infectadas. Tais medidas incluem o isolamento de casos; o incentivo à higienização das mãos, à adoção de etiqueta respiratória e ao uso de máscaras faciais caseiras; e medidas progressivas de 
distanciamento social, com o fechamento de escolas e universidades, a proibição de eventos de massa e de aglomerações (Aquino et al., 2020).

Estratégias de distanciamento social devem ser fortalecidas e realizadas de forma intersetorial e coordenada entre as diferentes esferas governamentais e regiões, para que seja alcançadoo fim da pandemia o mais brevemente possível e evitadas ondas de recrudescimento do contágio da doença (Aquino et al., 2020). P a r a t a n t o , necessita-se da conscientização da população, para que permaneça em casa, até a completa proibição da circulação nas ruas, exceto para compra de alimentos e medicamentos ou busca de assistência à saúde (Aquino et al., 2020).

Nesse contexto de incertezas, os indivíduos foram forçados a alterar a rotina e os laços sociais,para reduzir a velocidade de dispersão do vírus e, consequentemente, do número de pessoas que, simultaneamente, precisariam da estrutura hospitalar de alta complexidade para o tratamento dos sintomas graves (Grisotti, 2020). Essa estratégia é eficaz para evitar o colapso na assistência hospitalar e reduzir o número de vítimas da Covid-19 (Bezerra, 2020).

O isolamento social é uma das principais medidas não farmacológicas para o enfrentamento da pandemia (Garcia \& Duarte, 2020). De acordo com Farias (2020), pode não ser eficaz a médio prazo, devendo ser encarado, sobretudo, como uma medida para ganhar tempo enquanto cientistas do mundo estão à procura de tratamento e cura da Covid-19, pois a vacina exige mais experimentação (Farias, 2020).

\subsection{Alimentação e higiene durante o isolamento social}

A Covid-19 não é a primeira pandemia no a nível mundial e, certamente, não será a última. Contudo, será a primeira na qual, muito provavelmente, não haverá diminuiçãoda capacidade de distribuição alimentar e, consequentemente, não haverá escassez de alimentos (Oliveira, 2020).

O distanciamento fez rever algumas prioridades. Cozinhando em casa, passando mais tempo em família, lendo mais livros, assistindo mais filmes, ouvindo mais músicas, limpam-se as casas repetidamente, por medo da contaminação ou tempo ocioso (Guenther, 2020). Ainda segundo Guenther (2020), a pandemia mostrou que é possível trabalhar de casa. Muitos se sentem inclusive mais produtivos, pois economizam o tempo gasto de deslocamento de casa para o trabalho.

Malta et al. (2020), sobre o período de isolamento, discorrem que os brasileiros passaram a praticar menos atividade física, aumentaram o tempo dedicado às telas, reduziram o consumo de alimento saudáveis e intensificaram a ingesta de produtos ultraprocessados, em decorrência das restrições sociais impostas pela pandemia. Muitos fatores externos podem influenciar a imunidade e a saúde como um todo, uma alimentação saudável atua em conjunto para fortalecer o sistema imunológico e auxiliar na proteção de diversas doenças (Araújo et al., 2020). Ressaltando de que é sempre importante manter uma alimentação saudável e equilibrada, reduzindo o consumo energético excessivo e evitando o excesso de açúcar e sal (Costa et al., 2020). Os alimentos "in natura" ou minimamente processados possuem nutrientes e fitoquímicos com propriedades protetoras, antioxidantes e anti-inflamatórias, que melhoram a imunidade (Araújo et al., 2020).

Ainda de acordo com Costa et al. (2020), não existe alimento específico ousuplemento alimentar que possa prevenir ou ajudar no tratamento da Covid-19. As boas práticas de higiene continuam a ser a melhor forma de prevenção contra a doença.

O uso de EPI, como luvas e máscaras, é abordado nas regras de Boas Práticas de Manipulação e de Fabricação, entretanto, em perspectiva diferente, tendo como foco a redução da contaminação de alimentos (ANVISA, 2020). Ainda sobre o uso de máscaras, conforme a ANVISA (2020), apesar de o uso de máscaras não ser obrigatórioda legislação sanitária vigente no contexto das Boas Práticas de Fabricação e Manipulação de alimentos, esse equipamento pode ser utilizado para evitar a contaminação de alimentos prontos para o consumo e também para proteger a saúde do trabalhador em situações específicas.

A Covid-19 deve ser semelhante ao de outros tipos de coronavírus da mesma família, portanto, pode persistir por poucas horas ou vários dias, dependendo do tipo de superfície, temperatura e umidade do ambiente. Entretanto, os vírus são eliminados 
pela higienização ou desinfecção das superfícies (tanto pela ação de detergentes, sabões e desinfetantes) e pela lavagem das mãos (ANVISA, 2020).

Pandemias ocorreram ao longo da história e ocasionaram profundas rupturas no sistema social. O que se aprende com a história social das pandemias? Apesar do avanço científico e tecnológico dos últimos séculos, dispõem-se apenas, em termos práticos e no curto prazo, de dispositivos de biossegurança medievais (Grisotti, 2020).

\section{Metodologia}

Diante do cenário de agravamento da pandemia de covid-19 no brasil, muitas mudanças significativas ocorreram na vida e nos hábitos diários das pessoas, devido a isso, realizou-se pesquisa de forma on-line, para descobrir o que mudou realmente durante o período de isolamento social, no que se refere aos hábitos de higiene pessoal utilizados no combate à Covid-19, analisando se estão sendo cumpridas as orientações dos órgãos de saúde. Diante do cenário de isolamento social, imposto aos brasileiros durante a pandemia da Covid-19, faz-se necessário cuidar ainda mais da saúde e da imunidade (Araújo et al., 2020).

Para realização desta pesquisa, foi realizado estudo qualitativo sem definição de público-alvo específico, dado o período de pandemia, apresentando risco mínimo de participação. As informações obtidas através do questionário virtual elaborado nesta pesquisa foram utilizadas apenas para fins de pesquisa, não havendo dos participantes nada que causasse qualquer constrangimento uma vez que apenas dados como a cidade, faixa etária, grau de escolaridade foram solicitadas quanto a dados pessoais.

Foi elaborado um questionário com 19 questões, tendo como vantagens, segundo Pereira et al. (2018) a possibilidade de alcançarmos um grande número de participantes e desta forma podemos garantir o anonimato das respostas e sem a influência de opiniões de quem está fazendo a entrevista. A coleta de dados foi realizada totalmente em ambiente virtual, utilizando-se de redes sociais para divulgação, no período de outubro a novembro de 2020. A coleta dos dados ocorreu de modo on-line, com o auxílio do programa Google Forms ${ }^{\circledR}$, em que se criou o questionário contendo dezenove perguntas objetivas e subjetivas, as quais abordavam assuntos referentes a hábitos alimentares, de higiene pessoal e higienização de alimentos adquiridos e consumidos durante o período de isolamento social, na pandemia da Covid-19. A divulgação do formulário ocorreu utilizandose as redes sociais, mensagens através do WhatsApp ${ }^{\circledR}$ e Messenger ${ }^{\circledR}$ direcionadas a contatos dos autores/pesquisadores, para que respondessem ao questionário e repassassem para os outros contatos.

As informações foram extraídas para uma planilha CSV (Excel) e emitido um relatório (DASHBOARD) com todos os dados repassados da pesquisa, desta forma foi avaliado o comportamento de cada participante frente aos novos hábitos da população em época de pandemia. Com todos os dados consolidados e devidamente tratados, efetuou-se uma análise detalhada sobre o assunto estudado.

\section{Resultados e Discussão}

Conforme a Tabela 1, constatou-se que mais de 55\% dos participantes foram da região do Vale do Taquari-RS. O município de Encantado (RS) foi o que apresentou o maior número de participantes, 48 (29\%), isto se deve ao ciclo de amizades nas redes sociais onde a pesquisa foi divulgada. 
Tabela 1 - Participantes do estudo por cidade.

\begin{tabular}{lclc}
\hline \multicolumn{1}{c}{ Cidades } & $\mathrm{N}^{\mathrm{o}}$ de Participantes & \multicolumn{1}{c}{ Cidades } & $\mathrm{N}^{\mathrm{o}}$ de Participantes \\
\hline Angra dos Reis (RJ) & 1 & Laurentino (SC) & 2 \\
\hline Arroio do Meio (RS) & 4 & Marques de Souza (RS) & 2 \\
\hline Bento Gonçalves (RS) & 1 & Mogi das Cruzes (SP) & 1 \\
\hline Bom Retiro do Sul (RS) & 1 & Mossoró (RN) & 24 \\
\hline Brasília (DF) & 1 & Muçum (RS) & 1 \\
\hline Campinas (SP) & 1 & Nova Petrópolis (RJ) & 1 \\
\hline Canoas (RS) & 2 & Nova Prata (RS) & 1 \\
\hline Caxias do Sul (RS) & 3 & Nova Roma do Sul (RS) & 1 \\
\hline Chapecó (SC) & 2 & Porto Alegre (RS) & 2 \\
\hline Doutor Ricardo (RS) & 1 & Relvado (RS) & 1 \\
\hline Encantado (RS) & 48 & Roca Sales (RS) & 1 \\
\hline Estrela (RS) & 2 & Santa Maria (RS) & 1 \\
\hline Feliz (RS) & 1 & Santana do Livramento (RS) & 1 \\
\hline Garibaldi (RS) & 2 & Santo André (SP) & 1 \\
\hline General Câmara (RS) & 1 & São Leopoldo (RS) & 6 \\
\hline Gravatal RS) & 1 & Serrafina Correa (RS) & 1 \\
\hline Guaporé (RS) & 16 & Teutônia (RS) & 1 \\
\hline Itajaí (SC) & 1 & Uberlândia (MG) & 2 \\
\hline Jundiaí (SP) & 2 & Viamão (RS) & \\
\hline Lajeado (RS) & 9 & Westfália (RS) & \\
\hline & & Total: 165 & \\
\hline
\end{tabular}

Fonte: Autores (2021).

A maioria dos entrevistados foi do sexo feminino, 110 respostas (66,7\%), e 33,3\% foram do sexo masculino. As idades dos entrevistados foram: até 18 anos (6,1\%), de 19 a 29 anos (40,6\%), de 30 a 40 anos (25,5\%), de 41 a 50 anos (15,2\%) e, finalmente, maior que 51 anos (12,7\%). O público com idades entre 19 e 40 anos foi o que mais respondeu, este grupo representou $60 \%$ dos participantes que informaram ter escolaridade de nível superior completo ou incompleto ou pós-graduação. Quanto à escolaridade dos entrevistados, 32,1\% afirmaram possuir ensino médio completo, 20,6\% ensino superior incompleto e pósgraduação, 19,4\% ensino superior completo e, por fim, apenas 7,3\% relataram concluir o ensino fundamental.

Quanto à distribuição familiar dos entrevistados, cerca de $63 \%$ dos entrevistados disseram possuir na família até três pessoas, $25,5 \%$ até quatro, 4,8\% até cinco e, por fim, mais de cinco pessoas totalizaram 6,7\% dos entrevistados.

Questionou-se sobre os sintomas da Covid-19 entre os participantes e, ao analisar a Figura 1, a maioria absoluta dos entrevistados, 108 pessoas $(65,45 \%)$, não apresentou nenhum sintoma. Ainda de acordo com a pesquisa, 114 pessoas $(69,1 \%)$ informaram não precisar ficar em isolamento, deste modo, continuaram com a rotina. Contrariando o que constataram Aquino et al. (2020), a conjugação de isolamento dos casos, a quarentena de contatos e as medidas amplas de distanciamento social, principalmente aquelas que reduzem em pelo menos $60 \%$ os contatos sociais, têm o potencial de diminuir a transmissão da doença. Entre os sintomas mais comuns sentidos pelos entrevistados, informaram febre, tosse seca, dor de garganta e perda de paladar, os resultados demonstram que provavelmente não se tratavam de Covid-19.

Gonçalves e Toriani (2021), em pesquisa realizada com 120 estudantes entre 18 e 59 anos de uma instituição de ensino superior privada de Joinville (SC), destacaram que 57,5\% (n=69) obtiveram algum sintoma da Covid-19, como náuseas, vômitos, diarreia, falta de apetite e febre que acreditaram ter sido por consumo de alimento contaminado. 
Figura 1 - Classificação dos entrevistados quanto aos sintomas relatados.

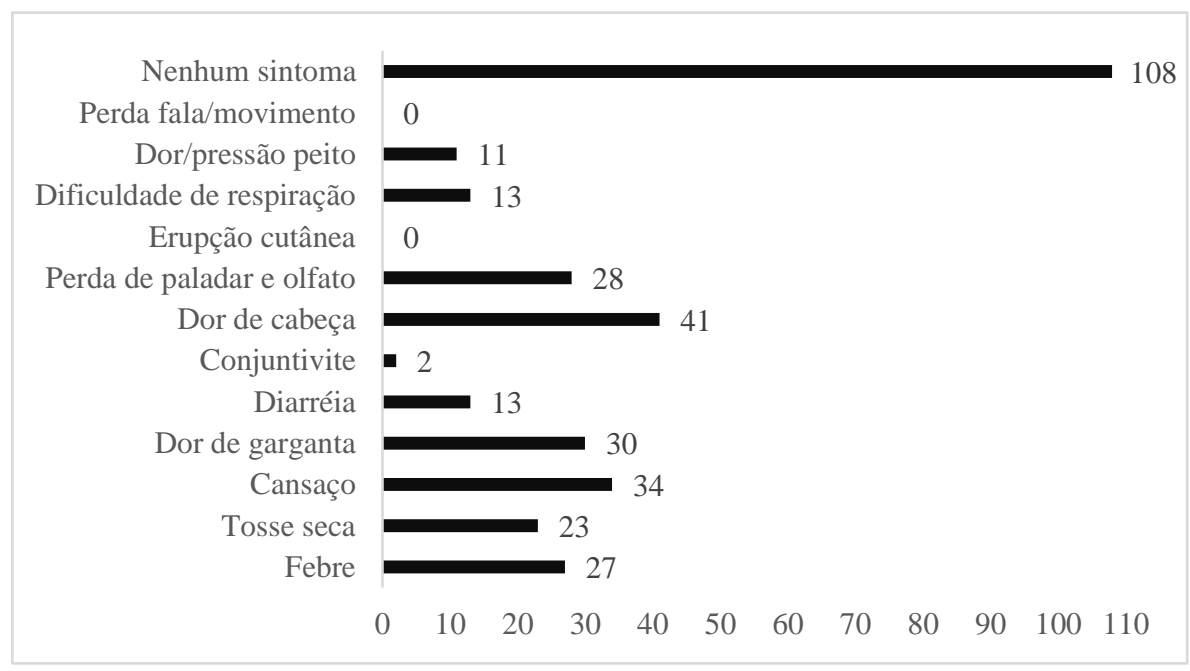

Fonte: Autores (2021).

A procura por hospitais pelos entrevistados foi baixa, duas pessoas relataram que precisaram de algum cuidado hospitalar, demonstrando a necessidade de maior atenção ao isolamento social. Concordando com a afirmação de Aquino et al. (2020) de que o isolamento social tem como principal objetivo restringir o contato entre as pessoas, buscando reduzir as chances de contaminação pelo vírus, a procura pelos serviços de saúde e o número de mortes. Porém, 114 entrevistados $(69,1 \%)$ informaram que não precisaram de nenhum cuidado especial e, por último, 49 pessoas $(26,7 \%)$ tiveram cuidados domiciliares.

Indagou-se, também, aos participantes do estudo sobre quais das etiquetas comportamentais orientadas pela ANVISA eram utilizadas. Pode-se observar na Tabela 2, que apesar da divulgação em meios de comunicação, o uso de máscara foi apontado por apenas 60,6\% dos entrevistados. A Lei 14.019, de 2 de junho de 2020 (Brasil, 2020), artigo $3^{\circ}$, relata que é obrigatório manter boca e nariz cobertos por máscara de proteção individual, conforme a legislação sanitária e na forma de regulamentação estabelecida pelo Poder Executivo Federal, para circulação em espaços públicos e privados acessíveis ao público, em vias públicas e transportes públicos coletivos.

Apenas 52,1\% os entrevistados responderam que higienizavam as mãos, evitavam aglomeração, cuidavam ao tossir, correspondendo a 35,2\% e 37,6\%, respectivamente. Evitar permanência em locais não arejados foi informado por 22,4\% e 51,5\% dos entrevistados relataram que seguiam todas as etiquetas recomendadas pela ANVISA (Tabela 2). Conforme Portaria $\mathrm{n}^{\circ} 319$ de 01/06/2020 (Secretaria da Saúde do Rio Grande do Sul, 2020), parágrafo II, é preciso orientar funcionários, colaboradores e usuáriosacerca da necessidade de higienização periódica das mãos, etiqueta respiratória (ao tossir ou espirrar usar o cotovelo flexionado ou lenço descartável e após higienizar as mãos) e distanciamento mínimo, bem como observar o cumprimento dessas medidas. 
Tabela 2 - Relação de resposta, conforme uso das etiquetas respiratórias e uso da máscara para manipular alimentos.

\begin{tabular}{lc}
\hline \multicolumn{1}{c}{ Etiquetas respiratórias da Anvisa } & $N^{o}$ de participantes \\
\hline Nenhuma & 3 \\
Todas & 85 \\
Uso máscara & 100 \\
Permanece em locais arejados & 37 \\
Evita aglomerações & 58 \\
Higienização das mãos & 86 \\
Medidas ao tossir/espirrar & 62 \\
& \\
\hline \multicolumn{1}{c}{ Quanto ao uso da máscara } & $N^{\circ}$ de participantes \\
\hline Usa máscara ao manipular alimentos & 48 \\
Não usa máscara ao manipular alimentos & 117 \\
Retira máscara ao falar & 19 \\
Não retira máscara ao falar & 146 \\
\hline
\end{tabular}

Fonte: Autores (2021).

Os resultados da Tabela 2 confirmam que a maioria das pessoas mantinha a máscara quanto falavam com outra $(88,5 \%)$, o que prova a preocupação dos participantes a respeito da proliferação do vírus. A pesquisa demonstrou que mais de $70 \%$ dos entrevistados não utilizavam máscara ao manipular alimentos. Esse dado revela que as pessoas necessitam ser orientadas quanto à importância da máscara nessa tarefa, pois o grande vetor é a saliva e a máscara cirúrgica ou caseira evita essa dispersão. Segundo o Ministério da Saúde, pesquisas têm apontado que as máscaras caseiras impedem que gotículas expelidas pelo nariz e bocas se espalhem pelo ambiente (Anvisa, 2020).

Questionou-se também sobre quais momentos os entrevistados higienizavam as mãos. Ao analisar a Figura 2, nota-se que as pessoas possuíam a preocupação em lavar as mãos, principalmente após irem ao sanitário e antes de comer. Ainda de acordo com os resultados, a maioria utilizava sabão ou sabonete, sendo o álcool em gel ainda utilizado por pouco mais de 17\% dos participantes, considerado, portanto, percentual insatisfatório, cujo uso foi popularizado devido à pandemia. Houve aumento do consumo dos produtos necessários ao enfrentamento à Covid-19, como álcool em gel, avental descartável, luvas de procedimento, máscara, óculos de proteção e touca descartável (Saraiva et al., 2020). 
Figura 2 - Periodicidade e métodos de higienização das mãos por participantes.

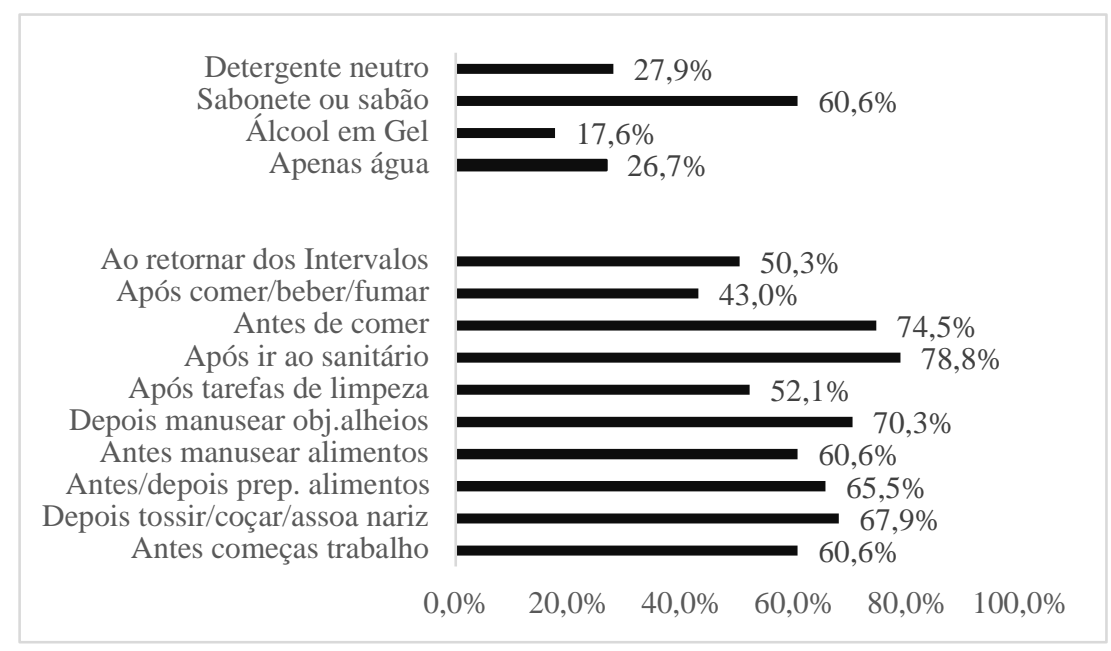

Fonte: Autores (2021).

Conforme as recomendações de higiene alimentar descritas pela OMS (Organização Mundial da Saúde), pela Fundação Oswaldo Cruz e Conselho Federal de Nutricionistas, é importante lavar as mãos com água e sabão com maior frequência, higienização de superfícies com álcool $70 \%$ ou água e sabão, antes e após o preparo dos alimentos, higienização dos alimentos embalados com água e sabão ou álcool 70\%. Frutas, verduras e legumes podem ser higienizados imersos por quinze minutos em água sanitária diluída em água (uma colher de sopa de água sanitária para cada litro de água) e enxaguados em água corrente. As embalagens de alimentos provindos de serviços de entrega em casa devem ser descartadas e, logo após, realizar a higienização das mãos (Fundação Oswaldo Cruz [FIOCRUZ] 2020; World Health Organization [WHO] 2020; Conselho Federal de Nutricionistas [CFN]; 2020).

As práticas adotadas de higiene pessoal durante a pandemia, de conservação e manipulação de alimentos ganharam evidência. Contudo, salienta-se que tais cuidados previnem não somente a contaminação pela Covid-19, como também contra Doenças Transmitidas por Alimentos (DTA) (Lima et al., 2020).

No estudo de Gonçalves e Toriani (2021), com relação a lavagens de mãos, 94,2\% dos participantes (n=113) afirmaram ter aumentado a frequência de lavagens de mãos por dia, após o início da pandemia, e 90,8\% (n=109) acreditavam que lavavam as mãos de forma válida. Além de prevenir a contaminação pela Covid-19, a lavagem correta das mãos é considerada de grande importância para evitar a propagação de DTA, quando há a incorreta higienização pessoal, pois, pode haver contaminação cruzada (Ávila et al., 2016).

Um percentual de 54,5\% dos entrevistados não costumavam higienizar as embalagens dos alimentos adquiridos no comércio, desconhecendo que os mesmos podem conter o vírus da Covid-19. A informação é fundamental para a população, pois com a divulgação desses hábitos de higiene, colabora-se com a prevenção e redução da vulnerabilidade das pessoas (Farias, 2020).

Gonçalves e Toriani (2021) relatam também que 68,3\% (n=82) dos estudantes não realizavam a higienização de alimentos e embalagens antes do início da pandemia do novo coronavírus. E, após o início da pandemia, 74,2\% (n=89) dos estudantes afirmaram higienizar alimentos e embalagens, destes, 53,9\% ( $\mathrm{n}=48)$ continuaram higienizando, mesmo após mais de três meses do início da pandemia, 23,6\% $(\mathrm{n}=21)$ relataram higienizar apenas quando lembravam e 22,5\% (n=20) informaram que após este período de mais de três meses após a pandemia, pararam de higienizar alimentos e embalagens de alimentos. O vírus causador da Covid-19 necessita de um hospedeiro humano ou animal para reprodução e, até o momento, não há na literatura relato ou evidência científica de contaminação do novo coronavírus por meio da alimentação (Anvisa, 2020). 
Observa-se, na Tabela 3, que 64,2\% das pessoas utilizavam apenas água para higienizar verduras e legumes, nota-se que poucas utilizavam algum produto para higienizá-las, a informação quanto à eficiência desses é muito importante e vem ao encontro do que constataram Costa et al. (2020), em que é preciso orientar as pessoas acerca das necessidades de utilizar hipoclorito de sódio, água sanitária, na proporção correta, pois estes produtos removem os microrganismos presentes em frutas e verduras.

Tabela 3 - Métodos de higienização de embalagens, verduras e legumes.

\begin{tabular}{lcc}
\hline \multicolumn{1}{c}{ Métodos de higienização } & $\begin{array}{c}\mathrm{N}^{\mathbf{o}} \text {. participantes } \\
\text { embalagens }\end{array}$ & $\begin{array}{c}\mathrm{N}^{\circ} \text {. participantes } \\
\text { verduras e legumes }\end{array}$ \\
\hline Somente água & 15 & 106 \\
\hline Água sanitária & 0 & 1 \\
\hline Álcool & 29 & 0 \\
\hline Às vezes & 2 & 41 \\
\hline Hipoclorito de sódio & 16 & 4 \\
\hline Detergente & 22 & 0 \\
\hline Desinfetante de superfícies & 1 & 1 \\
\hline Não compro & 0 & 0 \\
\hline Não higienizo embalagens & 90 & 1 \\
\hline Sabão em barra & 1 & 2 \\
\hline Sal, bicarbonato de sódio & 0 & 27 \\
\hline Vinagre & 6 & \\
\hline
\end{tabular}

Fonte: Autores (2021).

Ao ponderar as regras de isolamento social, mais de 152 entrevistados (70,37\%) afirmaram ter adquirido os alimentos durante a pandemia, indo pessoalmente ao mercado; 31 entrevistados (14,35\%) informaram que preferiam adquirir os alimentos através de delivery, entrega da mercadoria na casa do cliente, por motoboy ou carro de aplicativos locais. Já 19 pessoas $(8,79 \%)$ relataram que preferiam realizar as compras on-line, através da internet e, por fim, 14 pessoas optavam por comprar pelo uso de telefone. Em uma situação de isolamento social, o planejamento das compras se torna necessário para assegurar a oferta de alimentos, bem como o deslocamento, para que essa ação seja rápida e simples (Costa et al., 2020). Comprar alimentos de forma remota ainda não é comum, pois as pessoas ainda preferem o método tradicional de comprá-los.

Soares e Lima, (2020) destaca que se deve ter a atenção redobrada com a entrega de alimentos por delivery, pois o contato com o entregador pode ser um vetor do vírus, assim é aconselhado que o pagamento seja realizado pelo site ou aplicativo do estabelecimento. Após o recebimento das mercadorias, recomenda-se a higienização das mãos antes do recebimento e ao receber os alimentos, e deve-se manter distância mínima com o entregador de um metro (Soares \& Lima, 2020).

Quanto à higienização das mercadorias recebidas, recomenda-se que quando estes entrarem nas dependências da residência, o indivíduo higienize as mãos e retire o alimento da embalagem original, transferindo-o para o recipiente limpo e, em seguida, deve-se fazer novamente a higienização com água e sabão. Seguidamente, deve-se também descartar adequadamente a embalagem externa do alimento no lixo e higienizar a embalagem do alimento ou do produto com álcool em gel $70 \%$, posteriormente, higienizar as mãos com água e sabão ou álcool em gel 70\% (Soares e Lima 2020, conforme citado em CEREST, 2020).

Os participantes declararam que o tradicional jantar fora de casa aos finais de semana fez muita falta (47\%), conforme Figura 3. Ademais, 35\% dos entrevistados revelaram preferir ficar em casa. É preferível valorizar as refeições feitas em casa e aproveitar melhor os alimentos (Guenther, 2020).

O tradicional rodízio de pizza é hoje o preferido da maioria dos participantes, desbancando serviços tradicionais como rodízio de churrasco e buffets. 
Figura 3 - Refeições e serviços alimentares que fizeram falta no período de isolamento.

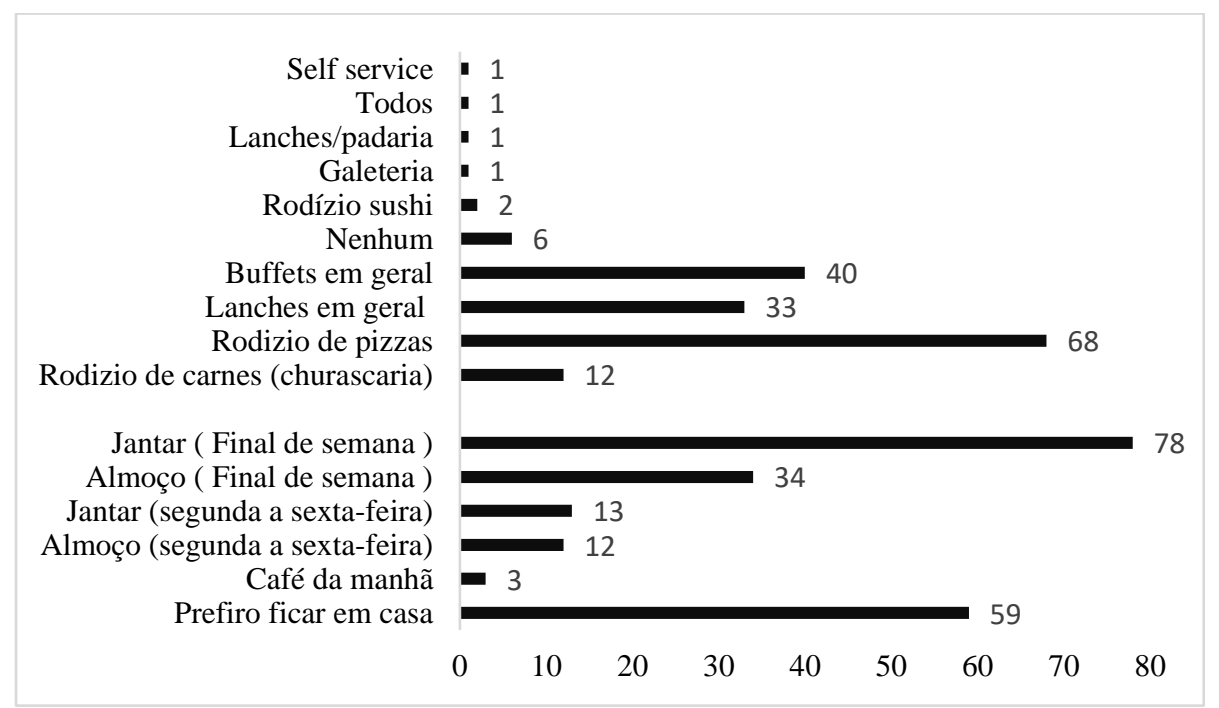

Fonte: Autores (2021).

Ao analisar a Tabela 4, 73,9\% dos participantes declararam ter consumido frutas. Mas, em contrapartida, a maioria absoluta consumiu produtos de alto valor energético, o que também foi constatado por Oliveira (2020), uma vez que elucidou em pesquisa que, em períodos de confinamento, há aumento da procura de alimentos de maior densidade energética, pobres em nutrientes essenciais e ricos em sal, gorduras e açúcares. Recomendam-se sempre alimentos in natura ou minimamente processados, ou seja, água, leite, iogurtes e frutas, no lugar de refrigerantes e biscoitos recheados, além de comida feita na hora e descartem-se produtos que dispensem preparação culinária (Araújo et al., 2020).

Tabela 4 - Relação de alimentos consumidos durante o isolamento.

\begin{tabular}{lcc}
\hline Alimentos & $N^{\circ}$ de paricipantes & $\%$ \\
\hline Salgadinhos em geral & 52 & 31,50 \\
\hline Bolachas, biscoitos & 56 & 33,90 \\
\hline Sanduíches & 103 & 62,40 \\
\hline Bolos, tortas, doces & 63 & 38,20 \\
\hline Verduras e legumes & 58 & 35,20 \\
\hline Frutas & 122 & 73,90 \\
\hline Refrigerantes & 30 & 18,20 \\
\hline Sucos artificiais & 25 & 15,20 \\
\hline Sucos integrais & 27 & 16,40 \\
\hline Produtos diet e light & 12 & 7,30 \\
\hline
\end{tabular}

Fonte: Autores (2021).

Souza e Vieira (2020) avaliaram as mudanças nos hábitos alimentares de 21 praticantes de atividades físicas durante a quarentena da Covid-19 e verificaram que os participantes aumentaram o consumo de refrigerantes (9,5\%), fast-foods e alimentos ultraprocessados $(38,1 \%)$, além do aumento no volume das refeições $(4,8 \%)$, entretanto, os autores observaram algumas mudanças nos hábitos alimentares positivos, como o maior consumo de frutas e hortaliças (19\%). Entretanto, 28,6\% dos participantes não relataram mudanças na alimentação.

Os autores Górnicka et al. (2020), na Polônia, em pesquisa com 2.381 indivíduos, buscaram identificar os padrões e as mudanças alimentares durante a pandemia e as respectivas associações no estilo de vida e verificaram que 43,3\% dos participantes diminuíram a frequência de atividades físicas e 34,3\% dos entrevistados aumentaram o consumo alimentar. 
Reyes-Olavarría et al. (2020), em estudo com 700 participantes chilenos para determinar as mudanças no estilo de vida causados pelo confinamento das pessoas no período de pandemia, concluíram que 45,3\% dos participantes afirmaram que consumiam média de três a cinco copos de água por dia; 51,3\% dos entrevistados mantiveram os hábitos alimentares anteriores à pandemia, entretanto, relataram comer em maior quantidade, além de 30\% relatarem manter o consumo de álcool diariamente. Quanto ao consumo de fast-foods, 62,9\% afirmaram que consumiam pelo menos de uma a duas vezes na semana. No tocante às atividades físicas, os participantes relataram que diminuíram a frequência, sendo 51,2\% dos homens e $58,7 \%$ das mulheres.

Di Renzo et al. (2020), na Itália, realizaram pesquisa on-line com 602 participantes, em que analisaram a relação entre os hábitos alimentares e o estado emocional, sendo verificado que $44 \%$ dos participantes faziam dieta antes da pandemia, $14 \%$ controlaram a alimentação, 48,7\% comiam quando estavam ansiosos, 55,1\% comiam para se sentir melhor e 57,8\% se sentiam ansiosos com os hábitos alimentares atuais.

\section{Conclusão}

A máscara é um dos instrumentos mais importantes no combate ao novo coronavírus, mantendo a distância e utilizando a máscara, diminui-se significativamente a chance da doença se proliferar. A informação clara e precisa, com a intenção de proteger a saúde das pessoas, faz-se necessária. Não se pode transformar um problema de saúde pública em um ato político.

A maioria dos entrevistados afirmou não ter sentido sintoma da doença. Entre os infectados, os sintomas mais comuns foram tosse, febre, dor de cabeça e perda do paladar. Apenas $2 \%$ tiveram que procurar auxílio em hospitais. Apesar de ser considerada uma pandemia com um vírus de fácil transmissão, nota-se que a maioria dos entrevistados não foi infectada, isso não descarta a possibilidade de alguns destes serem pacientes assintomáticos.

As mãos constituem um dos maiores vetores de contaminações, por isso, higienizá-las, após ir ao sanitário ou antes de preparar alimentos, é de suma importância para evitar a contaminação, seja pela Covid-19 ou outro microrganismo. De acordo com a pesquisa, a maioria das pessoas ainda não possuía o hábito de higienizar frutas e verduras compradas de terceiros. A higienização de hortifruti é um dos passos necessários para se ter um alimento seguro.

Os entrevistados, em maioria absoluta, adquiriam os alimentos indopessoalmente ao mercado ou feira. Isso demonstra a necessidade desses locais montarem estrutura adequada, para que não se tornem o grande vetor da doença.

A preocupação deve se estender aos restaurantes e similares, pois nota-se que esses serviços fizeram muita falta, uma vez que fazem parte de programas familiares tradicionais. A pesquisa revelou que a dieta alimentar das pessoas, apesar de a maioria ter consumido frutas, foi baseada em produtos de alto valor calórico. A maioria declarou ter consumido doces, salgadinhos, sanduíches e refrigerantes.

Evidenciou-se que as pessoas ainda preferem consumir produtos processados, não aderem ao consumo de produtos mais saudáveis, confirmando a hipótese inicial deste trabalho, seja pela praticidade ou rapidez de preparo. Sugere-se que novos estudos sejam realizados com relação as mudanças de comportamentos das pessoas após o término da pandemia avaliando se novos comportamentos tenham surgido e ainda entendemos a necessidade de serem reforçados os cuidados de higiene ao adquirir, transportar e armazenar os alimentos vindos dos locais de comercialização.

\section{Referências}

Agência Nacional de Vigilância Sanitária. (2020). O novo coronavírus pode ser transmitido por alimentos? ANVISA. https://www.gov.br/anvisa/ptbr/assuntos/noticias-anvisa/2020/o-novo-coronavirus-pode-ser-transmitido-por-alimentos

Agência Nacional de Vigilância Sanitária. (2020). Nota Técnica no 47/2020/SEI/GIALI/GGFIS/DIRE4/ANVISA de 3 de junho de 2020. Uso de luvas e máscaras em estabelecimentos da área de alimentos no contexto do enfrentamento ao COVID-19. ANVISA. https://www.gov.br/anvisa/ptbr/assuntos/paf/coronavirus/arquivos/arquivos-regulamentos/6994json-file-1 
Araújo, L., Antonino, P., Chaves, C., \& Salgado, S. (2020). Como a alimentação pode melhorar a imunidade? dicas para uma alimentação saudável durante a quarentena. Universidade Federal de Pernambuco. https://repositorio.ufpe.br/bitstream/123456789/37415/1/Alimen ta\%C3\%A7\%C3\%A3o\%20e\%20imunidade.pdf

Ávila, M. D. O., Santos, P. H. D. S., Gois, F. N. D., Furtado, M. D. C., \& Reis, I. A. D. O. (2016). A importância do controle das condições microbiológicas e higiênico sanitárias na prevenção de doenças transmitidas por alimentos - uma revisão de literatura. Instituto Federal de Sergipe. https://repositorio.ifs.edu.br/biblioteca/handle/123456789/766

Bezerra, A. C. V., Silva, C. E. M. D., Soares, F. R. G., \& Silva, J. A. M. D. (2020). Fatores associados ao comportamento da população durante o isolamento social na pandemia de COVID-19. Ciência \& Saúde Coletiva, 25, 2411-2421. https://doi.org/10.1590/1413-81232020256.1.10792020

Brandão, M (2021). Brasil tem 20 mil novos casos de covid-19 em 24 horas. Agência Brasil. https://agenciabrasil.ebc.com.br/saude/noticia/2021-01/brasil-tem20-mil-novos-casos-de-covid-19-em-24-horas

Brasil. (2020). Lei 14.019 de 02 de julho de 2020. Altera a Lei ${ }^{\circ} 13.979$, de 6 de fevereirode 2020, para dispor sobre a obrigatoriedade do uso de máscaras de proteção individual para circulação em espaços públicos e privados acessíveis ao público [...]. Publicado no DOU de 08.09.2020. https://www.in.gov.br/en/web/dou/-/lei-n-14.019-de-2-de-julho-de-2020-276227423

Brasil. (2020). Portaria MS/GM n. 188, de 03 de fevereiro de 2020. Declara Emergência em Saúde Pública de importância Nacional (ESPIN) em decorrência da InfecçãoHumana pelo novo Coronavírus (2019-nCoV). Publicado no DOU de 04.02.2020. http://www.in.gov.br/web/dou/-/portaria-n-188-de-3-de-fevereiro-de2020-241408388

Conselho Federal de Nutricionistas. (2020). Recomendações do CFN: boas práticas para a atuação do nutricionista e do técnico em nutrição e dietética durante a pandemia do novo coronavírus (COVID-19). https://www.cfn.org.br/wp-content/uploads/2020/03/nota_coronavirus_3-1.pdf

Costa, B. M. D., Neves, D. K. O. D., Meloni, L. C., Cordeiro, S. A., Lima, T. P. B., Marinho, W. N., Xavier, J., \& Santos, M. (2020). Guia de orientações em relação à alimentação e exercício físico diante da pandemia da doença pelo Sars-Cov-2 (Covid-19). FIOCRUZ. http://www.direh.fiocruz.br/aplicativos/gconteudo/ata20200327082853.pdf

Di Renzo, L., Gualtieri, P., Cinelli, G., Bigioni, G., Soldati, L., Attinà, A., Bianco, F. F., Caparello, G., Camodeca, V., Carrano, E., Ferraro, S., Giannattasio, S., Leggeri, C., Rampello, T., Lo Presti, L., Tarsitano, M. G., \& De Lorenzo, A. (2020). Psychological aspects and eating habits during COVID-19 home confinement: results of EHLC-COVID-19 italian online survey. Nutrients, 12(7), 2152. https://doi.org/10.3390/nu12072152

Di Renzo, L., Gualtieri, P., Pivari, F., Soldati, L., Attinà, A., Cinelli, G., Leggeri, C., Caparello, G., Barrea, L., Scerbo, F., Esposito, E., \& De Lorenzo, A. (2020). Eating habits and lifestyle changes during COVID-19 lockdown: an Italian survey. Journal of translational medicine, 18, 1-15. https://doi.org/10.1186/s12967020-02399-5

Farias, H. S. D. (2020). O avanço da Covid-19 e o isolamento social como estratégia pararedução da vulnerabilidade. Espaço e Economia. Revista brasileira de geografia econômica. https://doi.org/10.4000/espacoeconomia.11357

Folha de São Paulo. (2020). Governo federal decreta estado de emergência para conter Coronavírus no Brasil. https://www1.folha.uol.com.br/equilibrioesaude/2020/02/governo-decreta-estado-de-emergencia-por-causa-de-surto-do-coronavirus.shtml

Fundação Oswaldo Cruz. (2020). Quais as orientações para higienização de alimentos para prevenir a Covid-19? FIOCRUZ. https://portal.fiocruz.br/pergunta/quais-orientacoes-para-higienizacao-de-alimentos-para-prevenir-covid-19

Garcia, L. P., \& Duarte, E. (2020). Intervenções não farmacológicas para o enfrentamento à epidemia da COVID-19 no Brasil. Epidemiologia e Serviços de Saúde, 29(2), e2020222. Epub. https://doi.org/10.5123/s1679-49742020000200009

Gonçalves, B. M., \& Toriani, S. D. S. (2021). Hábitos relacionados à higiene alimentar em tempos de COVID-19: uma pesquisa com estudantes de uma instituição de ensino superior privada de Joinville (SC). Brazilian Journal of Development, 7(2), 18799-18811. https://doi.org/10.34117/bjdv7n2-497

Górnicka, M., Drywień, M. E., Zielinska, M. A., \& Hamułka, J. (2020). Dietary and lifestyle changes during COVID-19 and the subsequent lockdowns among polish adults: a cross-sectional online survey PLifeCOVID-19 study. Nutrients, 12(8), 2324. https://doi.org/10.3390/nu12082324

Grisotti, M. (2020). Pandemia de Covid-19: agenda de pesquisas em contextos de incertezas e contribuições das ciências sociais. Physis: Revista de Saúde Coletiva, 30, e300202. https://doi.org/10.1590/S0103-73312020300202

Guenther, M. (2020). Como será o amanhã? O mundo pós-pandemia. Revista Brasileira De Educação Ambiental (RevBEA), 15(4), 31-44. https://doi.org/10.34024/revbea.2020.v15.10766

Lima, S. K. R., Pinho, L. X., Oliveira e Silva, M. D. G. D., \& Lima, E. D. C. (2020). Impactos da COVID-19 no controle higiênico-sanitário no setor alimentício. Brazilian Journal of Development, 6(7), 53414-53445. https://doi.org/10.34117/bjdv6n7-838

Malta, D. C., et al (2020). A pandemia da COVID-19 e as mudanças no estilo de vida dos brasileiros adultos: um estudo transversal, 2020. Epidemiologia e Serviços de Saúde, 29, e2020407. https://doi.org/10.1590/s1679-49742020000400026

Ministério da Saúde (2020). Painel Coronavírus. https://covid.saude.gov.br

Oliveira, G. L. A., et al (2020). Medidas de distanciamento social e mobilidade na América do Sul durante a pandemia por COVID-19: condições necessárias e suficientes? arXiv. https://europepmc.org/article/ppr/ppr268933

Oliveira, A. S. D. (2020). Hábitos alimentares de estudantes universitários em períodode contenção social. Faculdade de Ciências da Nutrição e Alimentação. Universidade do Porto. https://repositorio-aberto.up.pt/bitstream/10216/127947/2/409895.pdf 
Research, Society and Development, v. 10, n. 6, e13910615502, 2021

(CC BY 4.0) | ISSN 2525-3409 | DOI: http://dx.doi.org/10.33448/rsd-v10i6.15502

Pereira, A. S., Shitsuka, D. M., Parreira, F. J., \& Shitsuka, R. (2018). Metodologia da pesquisa científica. Ed. Santa Maria, RS: UFSM, NTE. https://www.ufsm.br/app/uploads/sites/358/2019/02/Metodologia-da-Pesquisa Cientifica_final.pdf

Pereira, R. C., Lira, C. C., \& Souza Júnior, M. F. D. (2020). Preocupação produtiva e improdutiva em tempos de isolamento social. Editora Universitária da UFRPE. http://ww2.codai.ufrpe.br/node/272

Pereira, M. D., et al (2020). A pandemia COVID-19, isolamento social, consequências na saúde mental e estratégias de enfrentamento: uma revisão integrativa. Research, Society and Development , 9 (7), e652974548. https://doi.org/10.33448/rsd-v9i7.4548

Portal G1. (2021). Brasil soma 196,5 mil mortos por Covid; média móvel é de 707 óbitospor dia.

https://g1.globo.com/bemestar/coronavirus/noticia/2021/01/04/casos-e-mortes-por-coronavirus-em-4-de-janeiro-segundo-consorcio-de-veiculos-deimprensa.ghtml

Portal G1. (2021). Mundo registra mais de 1,1 milhão de novos casos de Covid-19 em 2021, segundo Johns Hopkins. https://g1.globo.com/bemestar/coronavirus/noticia/2021/01/03/mundo-registra-mais-de-11-milhao-de-novos-casos-de-covid-19-em-2021-segundo-johnshopkins.ghtml

Reyes-Olavarría, D, et al (2020). Positive and negative changes in food habits, physical activity patterns, and weight status during COVID-19 confinement: associated factors in the Chilean population. International Journal of Environmental Research and Public Health, 17(15), 5431. https://doi.org/10.3390/ijerph17155431

Saraiva, E. M. S, et al(2020). Impacto da pandemia pelo Covid-19 na provisão de equipamentos de proteção individual. Brazilian Journal of Development, 6(7), 43751-43762. https://doi.org/10.34117/bjdv6n7-115

Schmidt, B., et al (2020). Impactos na saúde mental e intervenções psicológicas diante da pandemia do novo coronavírus (COVID-19). https://doi.org/10.1590/SciELOPreprints.58

Schuchmann, A. Z., et al (2020). Isolamento social vertical x isolamento social horizontal: os dilemas sanitários e sociais no enfrentamento da pandemia de COVID-19. Brazilian Journal of Health Review, 3(2), 3556-3576. https://doi.org/10.34119/bjhrv3n2-185

Secretaria da Saúde do Rio Grande do Sul. (2020). Portaria SES $n^{\circ} 319$ de 20/05/2020. Institui o protocolo deboas práticas para prevenção do novo coronavírus (covid-19) a serem cumpridas pelos estabelecimentos que prestam serviços de alimentação, com consumo no local, no âmbito do Estado do Rio Grande do Sul. https://saude.rs.gov.br/upload/arquivos/202006/02101816-319-republic-cevs.pdf

Soares, A. C. N., \& Lima, M. R. D. S (2020). Serviços de delivery alimentício e suas precauções em tempos da pandemia de SARS-COV-2 (Covid-19). Brazilian Journal of Health Review, 3(3), 4217-4226. https://doi.org/10.34119/bjhrv3n3-025

Souza, E. B. D., \& Vieira, B. B. T. (2020). Avaliação do consumo alimentar durante o COVID-19. JIM-Jornal de Investigação Médica, 1(2), 032-043. https://doi.org/10.29073/jim.v1i2.291

Universidade Aberta do Sistema Único de Saúde (2020). Organização Mundial de Saúde declara pandemia do novo Coronavírus. UNA-SUS. https://www.unasus.gov.br/noticia/organizacao-mundial-de-saude-declara-pandemia-de-coronavirus

World Health Organization. (2020). Advice for the public on COVID-19. https://www.who.int/emergencies/diseases/novel-coronavirus-2019/advice-for-public 